\title{
A wearable low-cost device based upon Force-Sensing Resistors to detect single-finger forces
}

\author{
Claudio Castellini and Vikram Ravindra
}

\begin{abstract}
In rehabilitation robotics it is highly desirable to find novel human-machine interfaces for the disabled, in particular to substitute or augment surface electromyography (sEMG), trying to keep at the same time its easiness of use, precision and non-invasiveness. In this paper we design and demonstrate one such device, based upon Force-Sensing Resistors (FSRs). An array of 10 FSRs was wrapped around the proximal section of the forearm of ten intact subjects engaged in pressing on an accurate force sensor with their fingers (this includes the rotation of the thumb). The FSRs would detect the forearm surface deformations induced by muscle activity; the signals provided by the FSRs were then matched to the recorded forces. The experimental results show that finger forces can be predicted using this device with the same accuracy obtained in literature using sEMG. The device, even as an academic prototype, weighs about 65 grams and costs around 50 EUR. Thus, it is remarkably light and cheap in comparison to standard sEMG electrode arrays.
\end{abstract}

\section{INTRODUCTION}

Machine learning methods are often tested in conditions which have little or no significance for real applications [20]. In rehabilitation robotics, especially as far as prosthetics control is concerned, there is considerable disappointment since the application of machine learning to surface electromyography (sEMG), although successful in laboratory conditions, has not yet become the paradigm in the clinical environment. Not surprisingly, the community is calling for a radical change of focus [10], pushing for the discovery and testing of novel peripheral human-machine interfaces (PMIs) to be used in place of sEMG or to augment its capabilites. Following this indication, recently, some novel PMIs, alternative to sEMG, have been explored, such as, e.g., ultrasound imaging [11], [19] and the detection of the deformation of the forearm due to muscular activity [21], [3], [22].

We hereby propose one further such investigation, aimed at showing how precise a PMI based upon cheap force sensors can be in comparison to sEMG. We demonstrate both theoretically and practically that a simple array of 10 Force-Sensing Resistors (FSRs), wrapped around a subject's forearm, can be used to effectively predict the forces required at each fingertip. Each single-finger force (including thumb rotation) is simultaneously predicted, avoiding the drawback of using classification - an improvement with respect to the traditional approaches, going in the direction of natural control [6], [16], [1].

The authors are with the Robotics and Mechatronics Center, DLR (German Aerospace Center), 82234 Oberpfaffenhofen, Germany. Vikram Ravindra is also with the Technical University of Munich, Germany. email: claudio.castellini@dlr.de

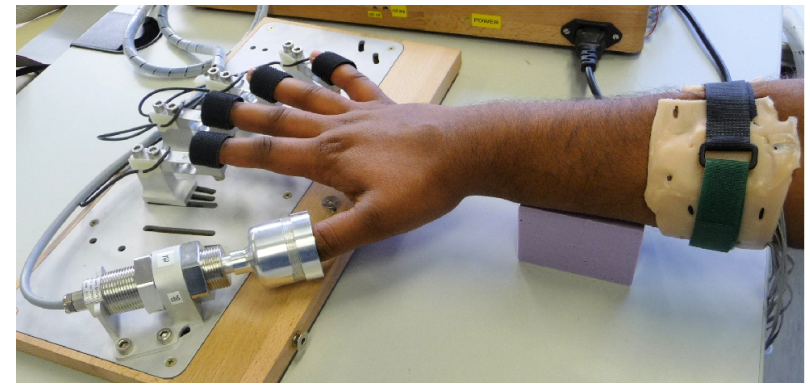

Fig. 1. A bird's eye view of the experimental setup: the subject's finger forces are measured by a strain-gauge-based high-precision force sensor (FFLS), while a bracelet fitted with Force-Sensing Resistors is wrapped around the forearm.

Surprisingly, our results show that this device can be used to obtain a precision of up to 1.5 Newtons in the prediction of the fingertip forces. Such a precision is comparable to that obtained with sEMG [13], [7]. The device is wearable, weighing about 65 grams, and costs as a prototype less than 50 EUR.

The movie provided as supplemental material shows the device in a live demonstration.

\section{Comparison with related work}

Human motion is due to muscular activity and the related torques and forces applied at the skeletal joints. Muscular activity implies the deformation and motion of the involved muscles (and all other musculoskeletal structures) inside the body, and this is in turn reflected in deformations of the surface of the body. These surface deformations could be in principle used to reconstruct the muscular activity - a task traditionally achieved using surface electromyography [14], [15]. Body deformations due to muscular activity, as an alternative to sEMG, have been widely explored in the pioneering works by, among others, Craelius and Wininger [4], [17], [21]: force sensors are tightened to the body surface against a rigid holder, so that their response reflects the inner deformations of the musculoskeletal structure. More recently, the technique has been successfully applied to a lightweight prosthetic hand [3]. In all these cases, classification has been applied to the force signals (historically called FMG, Force Myography, RKI, Residual Kinetic Imaging, or SMP, Surface Muscle Pressure) to detect which kind of action was intended by a human subject; furthermore, in [22], Extreme Learning Classification [9] was applied to a similar kind of signals to classify six different upper-limb motions.

The work proposed herewith advances the state of the art 


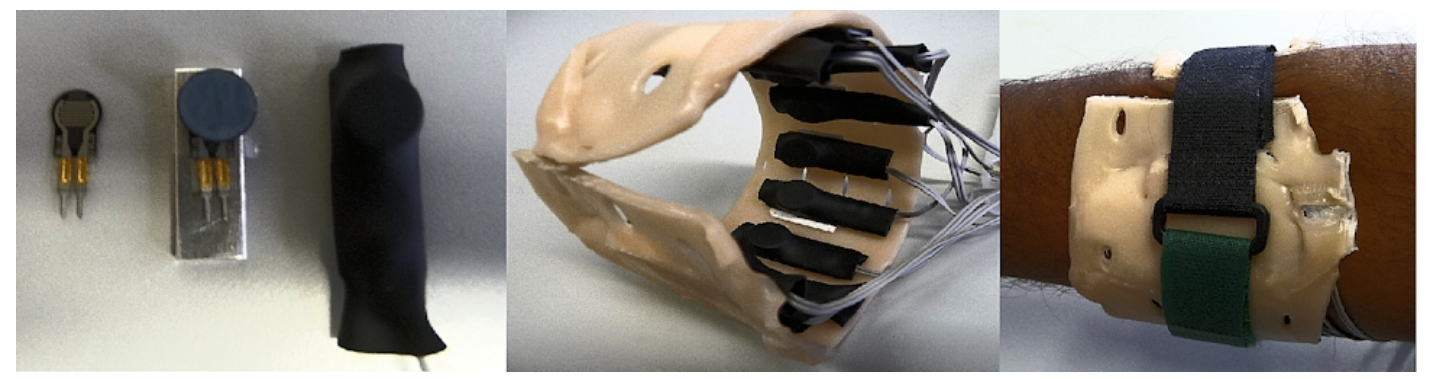

Fig. 2. Construction of the bracelet. (left) Different stages in the construction of the sensors. (middle) The bracelet, with 10 FSRs housed in it. (right) The bracelet strapped onto a forearm.

by showing how six finger forces can be simultaneously predicted, using an extremely simple regression method, and retaining all good properties of the already proposed approaches.

\section{EXPERIMENT DESCRIPTION}

\section{A. Experimental setup}

The setup consisted of a semi-rigid bracelet, in the interior of which 10 pressure sensors were housed; their signals were acquired using a standard analogic/digital conversion card. Moreover, exact finger forces were gathered using the FFLS, a strain-gauge-based multiple force sensor.

1) Force sensors: Ten Force-Sensing Resistors FSR400-short by Interlink Electronics (www.interlinkelectronics.com/FSR400short.php) were used to determine the forearm deformation. These sensors expose a $5.6 \mathrm{~mm}$-diameter sensitive area made of polymer thick film, whose electrical resistance decreases as the force applied to it increases. Although their force sensitivity range can be as large as $0.2 \mathrm{~N}$ to $40 \mathrm{~N}$ (and even more, as experimentally determined), their transfer function is typically non-linear, with no guarantee of repeatability across sensors, and a non-negligible hysteresis, for high force values. These characteristics justify their relatively low price: each FSR400-short is available in Germany at a single-unit price of less than 5 EUR.

Following the guidelines for optimal performance found in the user's manual of the FSR400, each sensor was affixed onto an aluminium plate of dimensions $3 \mathrm{~cm} \mathrm{x} 1 \mathrm{~cm} \mathrm{x} 1$ $\mathrm{mm}$ by means of a double sided adhesive tape, in order to provide a rigid surface on the back of the sensor. A semispherical rigid rubber "foot" was placed upside-down on top of the sensitive surface, in order to concentrate the force onto it. This customised arrangement was then encased in a heat shrink tubing of width $1.5 \mathrm{~cm}$; a hot-air gun was then used to shrink the tube to fit the shape of the sensor and keep its parts together. One sensor at the various stages of the procedure can be seen in Figure 2 (left).

2) Bracelet: A sheet of dimension $25 \mathrm{~cm} \mathrm{x} 5 \mathrm{~cm}$ of thermoplastic splinting material was cut in order to build the bracelet which would host the FSRs. The sheet was immersed in water at $60^{\circ} \mathrm{C}$ for about 20 seconds, then wrapped around the forearm of the experimenter and held firmly in position for one minute. A thin gap was left between the two ends so that the bracelet could be strapped with ease. Once the sheet cooled to room temperature, the bracelet retained the shape of a cast that was moulded to fit the forearm.

The force sensors described above were attached to the inner surface of the bracelet by means of a strong double sided adhesive tape (2, left); their placement was evenly spaced, so as to effectively gather signals from the entire surface of the forearm (Figure 2, right). Lastly, a hookand-loop strap was used to tighten the bracelet around the forearm.

The signal from each sensor was amplified by means of a custom-made operational amplifier connected (again, as per the user's manual of the sensors) to the ground via a variable resistor. Changing the value of the resistor changes the sensitivity of the sensors.

3) The FFLS: In order to reliably record the finger forces, we used a slightly modified version of the FFLS (FingerForce Linear Sensor [12]). The FFLS measures single-finger flexion and extension forces (finger flexion and extension refer to pushing the finger down and pulling the finger up respectively) of the index, middle, ring and little fingers, using an individually calibrated $\pm 20 \mathrm{~N}$ industrial straingauge-based force sensor for each finger. Thumb rotation and adduction/abduction forces are captured using a single radial dual-axis calibrated strain-gauge sensor with range of $\pm 40 \mathrm{~N}$. These sensors are characterized by their high signal repeatability, minimal drift over time and almost perfect linearity and non-existent hysteresis with both parameters guaranteed to deviate not more than $0.3 \%$. Each strain gauge force sensor is connected to a dedicated industrial strain gauge amplifier, which converts the applied force into an analog voltage.

4) Calibration: Given that the FSRs are not guaranteed to all have the same transfer function, a calibration phase was necessary. Each FSR in turn was firmly affixed on one of the strain-gauge sensors of the FFLS; this ensured that the pressure exerted on the FSR was recorded by both the FSR and the strain-gauge sensor, enabling us to relate the FSR values to the forces actually exerted. The strain gauge sensor, being extremely accurate and perfectly linear, serves as an excellent yardstick to measure the transfer functions of the FSRs. Pressure was repeatedly exerted on the FSR with a single finger with various speeds and pressures. 
Expecting very small forces to be exerted onto the FSRs by the forearm deformations, we tried to have the sensors work in the linear zone. An initial round of experiments revealed that these forces are in the range of 0-15 Newtons; we then set the resistors connecting the operational amplifiers to the ground in a way that the saturation point of the FSRs would happen slightly above 20 Newtons. Using these settings, the relationship between the pressure signals from the FSRs (denoted by $p$ ) and the force signals from the strain gauge sensor (denoted by $f$ ) turned out to be reliably approximated by a linear relationship $f=\alpha p+\beta$, with average values of $\alpha=-6.7299 \pm 0.3593$ and $\beta=22.6730 \pm 1.3186$, showing that the behaviour of each FSR is comparable. The average $\mathrm{R}$-squared coefficient across all sensors was found to be $0.98 \pm 0.01$. No measurable hysteresis effect was observed.

Figure 3 shows a typical calibration data collection, plus a linear fit. Such a behaviour of the FSRs is actually expected, since from the datasheet it is apparent that, for reasonably small forces, their transfer function is linear.

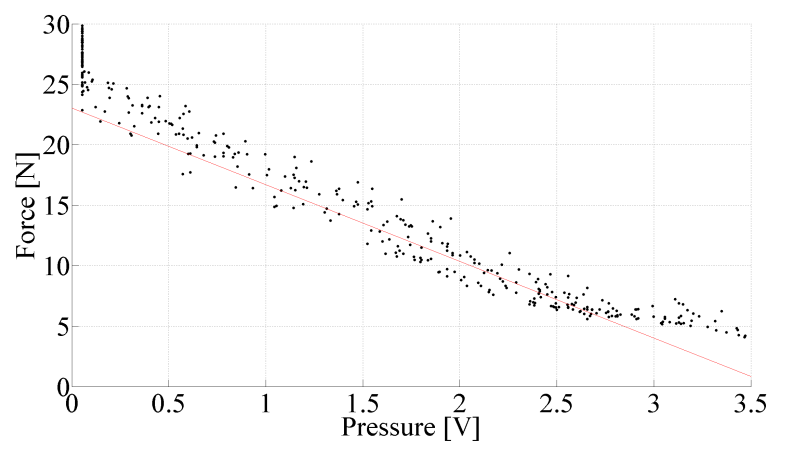

Fig. 3. Typical data obtained during the calibration of a FSR and a linear fit limited to the region of interest, that is, for output values larger than 0.1 Volts. The R-squared coefficient is 0.97 .

\section{B. Experimental Protocol}

Ten healthy able-bodied people participated in the experiment. The subjects were given a detailed oral and written description of the procedure. They affirmed their cognizance of the same and voluntary participation in an informed consent form. The experiment was approved by the Ethical Committee of the DLR.

Each subject sat comfortably on an adjustable office chair, maintaining an upright posture. The bracelet was affixed to the forearm such that it was firmly attached and was comfortable to the subject. The fingertips of the subject were placed over the sensors of the FFLSs and strapped to each sensor using a hoop-and-loop ring. The subject was then asked to apply forces according to a visual stimulus. A total of five "trials" were performed by each subject in a single session. In each trial, pressure was applied by the finger tips one at a time (little, ring, middle, index finger, thumb rotation and thumb adduction). The FSR data from the bracelet, the force data from the FFLS and the visual stimulus were recorded continuously and simultaneously during the entire procedure at $50 \mathrm{~Hz}$.

\section{Data Analysis}

An initial round of experiments revealed that filtering the input data and/or the output data would not significantly change the performance of the prediction; we therefore decided to employ the raw data for training, as this would entail a smaller delay in the prediction. A comparative analysis of three different machine learning methods was enforced, namely Ridge Regression (RR, [8]), Support Vector Regression (which we will denote as SVM, [2], [5]) and Ridge Regression with Random Fourier Features (denoted as RFF, [18]). RR is a regularised variant of Least-Squares Regression, intended as a very basic linear regression method; among its advantages, it is extremely fast and can easily be made incremental, a very desirable characteristic to be implemented in an online scenario. On the other hand, SVM employs a radial basis kernel to find a non-linear approximation and is therefore deemed to be more accurate than RR; it has however the disadvantage that it cannot run incrementally. Lastly, RFF is a non-linear extension to RR, approximating a radial basis kernel thanks to a hyperparameter dalled $D$, but retaining the speed and incrementality of RR.

On top of this, we tried three different ways of training and testing the above-mentioned approaches:

1) in the first scenario, 5-fold cross-validation on shuffled data for each subject was employed, and the full range of the force data recorded from the FFLS was used. This scenario would represent the optimal conditions obtained in a laboratory experiment;

2 ) in the second scenario, 5-fold cross-validation was enforced "trial-wise", that is, for each subect, we used four trials for training and one for testing. This scenario is expected to be harder than the first one, as no training data for the testing trial would be available;

3) lastly, in the third scenario, only the maximal and minimal values of the stimulus, and not of the FFLS sensors, were used to train.

In all cases, testing was done on the full range of the true values obtained from the FFLS sensors, in order to retain maximum realism. Notice that the last scenario in particular reflects the so-called "realistic" scenario already sucessfully employed for with ultrasound imaging and sEMG in [19], [7]. Its motivation lies in the fact that, in the typical application of this system, namely control of a hand prosthesis by a transradial amputee, no ground truth would be available in principle. The usage of "on-off" stimulus values as the ground truth represents therefore an approximation of the real case, in which no graded force values would ever possibly be available. It was expected to see a degradation in performance from scenario 1 to scenario 3 .

For each subject, grid-search was used to find the hyperparameters for each machine learning method, namely $D$ and the radial basis function's standard deviation $\sigma$ for RFF, and $C$ and $\sigma$ in the case of the SVM. (For more details on such hyperparameters, refer once again to [7].) As a measure of performance, we used the Root Mean Squared 
Error normalised over the target values range (nRMSE) for all scenarios; in the third scenario we also used the absolute RMSE expressed in Newtons, in order to have an idea of what the actual error in the worst case would be.

\section{RESULTS}

Figure 4 shows the experimental results, for all considered methods and averaged over all subjects. In the Figure, the first panel from the top shows the results obtained in scenario 1 (training and testing on FFLS data, cross-validation done with shuffling); the second panel shows the second scenario (cross-validation by training on four trials and testing on the remaining trial); and the third and fourth panels show the performance in the "realistic setting" (third scenario, training on on-off stimulus values), as well as the nRMSE and the absolute RMSE in Newtons. As it can be seen, in scenarios 1 and 2, RR always performs significantly worse (two-sample Student's t-test $p<0.01$ ) than the kernel-based approaches, with little or no difference between RFF and SVM. All finger forces are predicted with similar accuracy, the nRMSE ranging between less than $5 \%$ and $14 \%$.

In the third scenario (third and fourth panels of Figure 4), an error of up to 4 Newtons is observed (RR, thumb rotation), while the best performance is obtained on the ring finger by the RFF (about 1.5 Newtons). Notice, however, that the difference in performance between RR and the other approaches is sensibly lower than in the former two cases. Statistically significantly different performance (Student's test again) is observed in some of the cases, but not in all of them - for instance in the case of the middle finger, in which RFF and SVM do not outperform Ridge Regression.

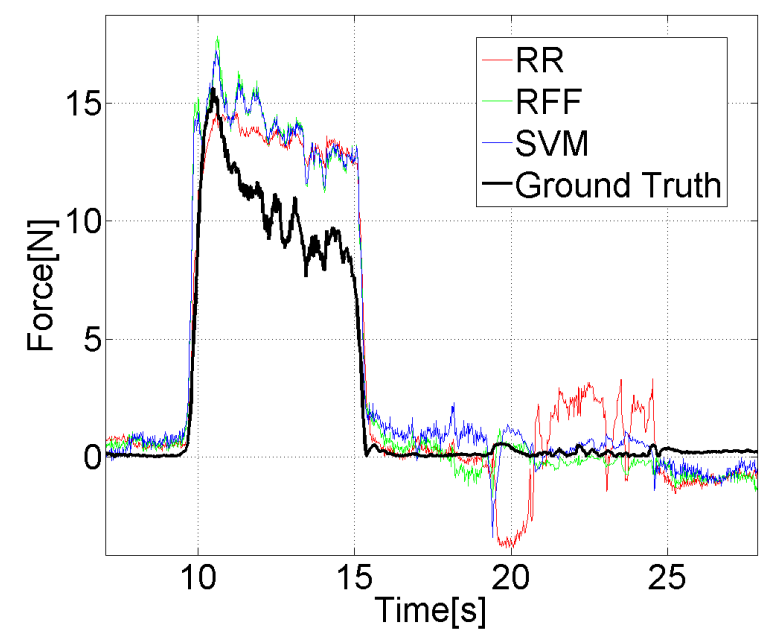

Fig. 5. Ground truth and predictions obtained by RR, RFF and SVM during a typical repetition in scenario 3 .

Figure 5 comparatively shows a typical prediction (index finger). As one can see, all prediction methods seem to perform similarly during the phase in which the finger is actively pressing (approximately from 9 to 16 seconds); and they seem not to take into account the natural effect of slightly releasing the pressure during the action. This is reasonable, since in this scenario the ground truth for training was the stimulus values and not the force values, and the stimulus constantly evaluates to 1 during the pressure. As opposed to that, during the phase in which the index is not active (in this graph, before 9 seconds and from 16 seconds on) RR performs badly if compared to SVM and RFF. This is related to the nature of linear regression, which in general cannot damp spurious changes in the input space values. Notice, moreover, that RFF performs very close to SVM, which is reasonable, since as a kernel-based method RFFs are a finite approximation of a Gaussian kernel.

\section{CONCLUSion AND Future Work}

In this paper we have described and demonstrated a wearable, low-cost device to detect fingertip forces. The device consists of a plastic bracelet fitted with ten ForceSensing Resistors; these sensors, whose characteristic curve is highly non-linear for high forces, are shown in this case to be working in the linear section of their operating range. Three machine learning methods have been applied to their signal in order to predict finger forces, namely Ridge Regression, Support Vector Regression and Ridge Regression with Random Fourier Features. The error obtained is less than one Newton, and is in the worst case of about 4 Newtons, making the accuracy comparable to that obtained by surface electromyography [7]. The best accuracy is about 1.5 Newtons.

The experimental results shown in this paper are to some extent surprising. They show that this device, weighing only about 65 grams, and whose total price (for a prototype) is about 50 EUR, can be used to predict finger forces to a remarkable accuracy by employing Random Fourier Features, a method already employed for sEMG [7]. The performance of FSR may clearly be worse in case of obese subjects, as the deformation on the surface of the forearm would be hindered; however, sEMG is also known to suffer from dampening of signals due to excessive fat tissue. This lets us hope that it can be used in the near future as an enhancement / replacement for sEMG. The immediate future work includes a direct comparative analysis with sEMG and ultrasound imaging, in order to prove the actual feasibility of the approach and to check the advantages and disadvantages of this novel PMI with respect to more traditional ones. Moreover, we plan to check how resilient to arm movements and wrist rotation such a prediction is, by using this device in a pick and place experiment, and with amputees.

\section{ACKNOWLEDGMENTS}

We are grateful to Dr. Risto Kõiva of the University of Bielefeld (Germany) for building and making available the FFLS v2.0.

\section{REFERENCES}

[1] A. Ameri, E. Scheme, E. Kamavuako, K. Englehart, and P. Parker. Real-time, simultaneous myoelectric control using force and positionbased training paradigms. Biomedical Engineering, IEEE Transactions on, 61(2):279-287, Feb 2014. 
Training: Ground truth(sensor values); Train/test partition: Random

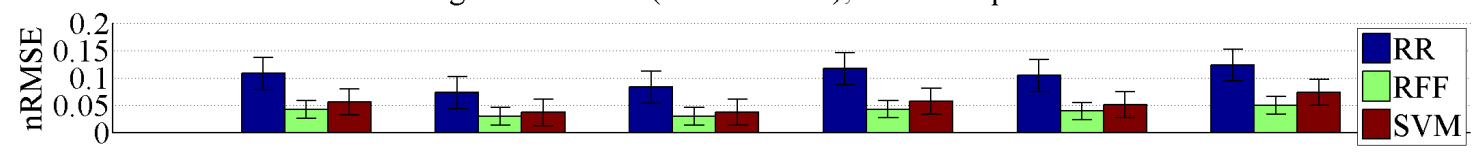

Training: Ground Truth(sensor values); Train/test partition: Trialwise

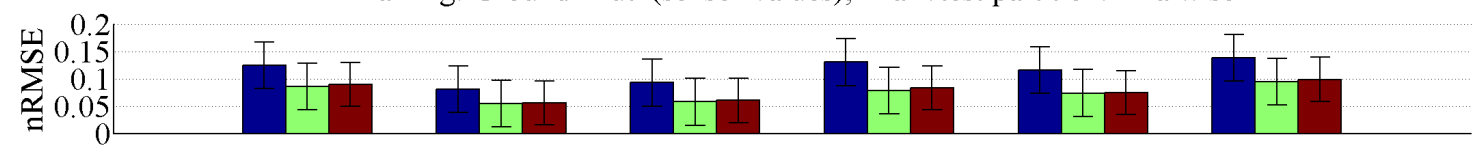

Training: Stimulus; Train/test partition: Trialwise

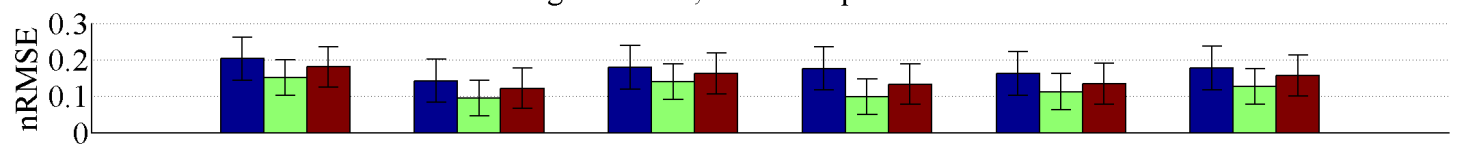

Training: Stimulus; Train/test partition: Trialwise

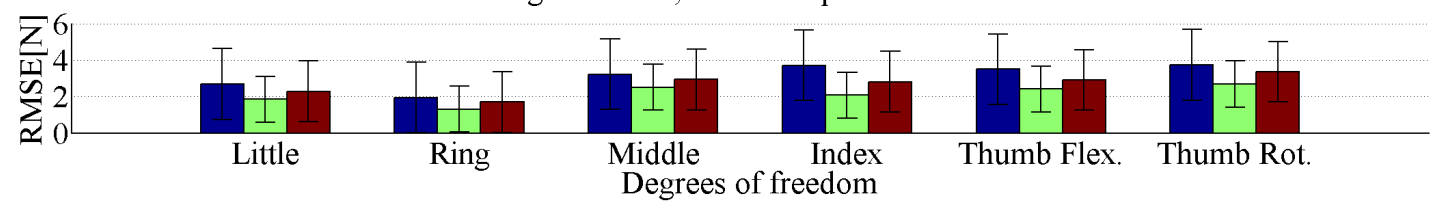

Fig. 4. nRMSE for the three machine learning methods considered (RR, RFF and SVM) in scenarios 1, 2 and 3 (first, second and third panels top to bottom); the fourth panel additionally shows the root mean-squared error expressed in Newtons, for the third scenario. Results for all subjects, average performance values over all subjects plus/minus one standard deviation.

[2] B. E. Boser, I. M. Guyon, and V. N. Vapnik. A training algorithm for optimal margin classifiers. In D. Haussler, editor, 5th Annual ACM Workshop on Computational Learning Theory (COLT), pages 144152, Pittsburgh, PA, USA, 1992. ACM press.

[3] N. Carbonaro, G. Anania, M. Bacchereti, G. Donati, L. Ferretti, G. Pellicci, G. Parrini, N. Vitetta, D. Rossi, and A. Tognetti. An innovative multisensor controlled prosthetic hand. In L. M. Roa Romero, editor, XIII Mediterranean Conference on Medical and Biological Engineering and Computing 2013, volume 41 of IFMBE Proceedings, pages 93-96. Springer International Publishing, 2014.

[4] W. Craelius. The bionic man: Restoring mobility. Science, 295(5557):1018-1021, 2002.

[5] N. Cristianini and J. Shawe-Taylor. An Introduction to Support Vector Machines (and Other Kernel-Based Learning Methods). CUP, 2000.

[6] A. Fougner, E. Scheme, A. D. C. Chan, K. Englehart, and O. Stavdahl. Resolving the limb position effect in myoelectric pattern recognition. IEEE Transactions on Neural Systems and Rehabilitation Engineering, 19(6):644-651, 2011.

[7] A. Gijsberts, R. Bohra, D. Sierra González, A. Werner, M. Nowak, B. Caputo, M. Roa, and C. Castellini. Stable myoelectric control of a hand prosthesis using non-linear incremental learning. Frontiers in Neurorobotics, 8(8), 2014.

[8] A. E. Hoerl and R. W. Kennard. Ridge regression: Biased estimation for nonorthogonal problems. Technometrics, 12:55-67, 1970.

[9] G.-B. Huang, Q.-Y. Zhu, and C.-K. Siew. Extreme learning machine: a new learning scheme of feedforward neural networks. In Neural Networks, 2004. Proceedings. 2004 IEEE International Joint Conference on, volume 2, pages 985-990 vol.2, July 2004.

[10] N. Jiang, S. Dosen, K. Muller, and D. Farina. Myoelectric control of artificial limbs: Is there a need to change focus? [in the spotlight]. IEEE Signal Processing Magazine, 29(5):152-150, 2012.

[11] G. Jing-Yi, Z. Yong-Ping, L. P. J. Kenney, A. Bowen, D. Howard, and J. J. Canderle. A comparative evaluation of sonomyography, electromyography, force and wrist angle in a discrete tracking task. Ultrasound in Med. \& Biol, 37(6):884-891, 2011.

[12] R. Kõiva, B. Hilsenbeck, and C. Castellini. Ffls: An accurate linear device for measuring synergistic finger contractions. In Proceedings of EMBC - the 34th annual conference of the IEEE Engineering in Medicine and Biology Society, pages 531-534, 2012.

[13] R. Kõiva, B. Hilsenbeck, and C. Castellini. Evaluating subsampling strategies for semg-based prediction of voluntary muscle contractions. In Proceedings of ICORR - International Conference on Rehabilitation Robotics, pages 1-7, 2013.

[14] R. Merletti, M. Aventaggiato, A. Botter, A. Holobar, H. Marateb, and T. Vieira. Advances in surface EMG: Recent progress in detection and processing techniques. Critical reviews in biomedical engineering, 38(4):305-345, 2011.

[15] R. Merletti, A. Botter, C. Cescon, M. Minetto, and T. Vieira. Advances in surface EMG: Recent progress in clinical research applications. Critical reviews in biomedical engineering, 38(4):347-379, 2011.

[16] S. Muceli, N. Jiang, and D. Farina. Extracting signals robust to electrode number and shift for online simultaneous and proportional myoelectric control by factorization algorithms. Neural Systems and Rehabilitation Engineering, IEEE Transactions on, 2013. to appear.

[17] S. L. Phillips and W. Craelius. Residual kinetic imaging: A versatile interface for prosthetic control. Robotica, 23(3):277-282, May 2005.

[18] A. Rahimi and B. Recht. Uniform approximation of functions with random bases. In Allerton Conference on Communication Control and Computing (Allerton08), pages 555-561, September 2008.

[19] D. Sierra González and C. Castellini. A realistic implementation of ultrasound imaging as a human-machine interface for upper-limb amputees. Frontiers in Neurorobotics, 7(17), 2013.

[20] K. Wagstaff. Machine learning that matters. In J. Langford and J. Pineau, editors, Proceedings of the 29th International Conference on Machine Learning (ICML-12), ICML '12, pages 529-536, New York, NY, USA, July 2012. Omnipress.

[21] M. Wininger, N.-H. Kim, and W. Craelius. Pressure signature of forearm as predictor of grip force. Journal of Rehabilitation Research and Development, 45(6):883-892, 2008.

[22] Z. Xiao and C. Menon. Towards the development of a wearable feedback system for monitoring the activities of the upper-extremities. Journal of NeuroEngineering and Rehabilitation, 11(1):1-13, 2014. 